Laboratốrio de Entomologia, Instituto Agronômico de Pernambuco (IPA), Av. General San Martin, 1371, Bongi, CEP 50761-000, Recife, PE Brasil

2Departamentos de Entomologia

Quîmica, Ciências Exatas, Universidade Federal de Lavras (UFLA), Lavras, MC, Brasil

* autor correspondente

\ cynthia.lacerda@ipa.br

\section{Avaliação da repelência de tomateiros ao ácaro rajado}

\author{
Evaluation of the tomato plants repellency to two-spotted \\ spider mite
}

Cynthia Araújo de Lacerda ${ }^{*}$, Américo Iorio Ciociola², Maria das Graças Cardoso², Custódio Donizete dos Santos², Ruben Delly Veiga²
RESUMO: O tomateiro é atacado por artrópodospragas, como o ácaro rajado Tetranychus urticae (Koch) (Acari: Tetranychidae). Este trabalho caracteriza os graus de repelência de genótipos de tomateiro a $T$. urticae. Realizaram-se na Universidade Federal de Lavras, de maio a novembro de 2001, com temperaturas médias de $23 \pm 2{ }^{\circ} \mathrm{C}$, quatro experimentos com 19 tratamentos $(\mathrm{T})$ e 3 repetições $(\mathrm{r}), 6 \mathrm{~T}$ e $3 \mathrm{r}$, $19 \mathrm{~T}$ e $3 \mathrm{r}$ e $16 \mathrm{~T}$ e $5 \mathrm{r}$. Os T (genótipos) foram: Testemunhas suscetíveis, Solanum lycopersicum cv. IPA-5 e S. lycopersicum cv. Caline IPA-6; resistentes, Lycopersicon hirsutum f. glabratum PI 134417 e L. hirsutum f. glabratum C. H. Mull.; progênies de $S$. lycopersicum x Lycopersicon hirsutum f. glabratum : $184.1 .1 \mathrm{~m} 2 ; 199.2 .2 \mathrm{~m} 2$; $206.1 .2 \mathrm{~m} 2 ; 213.1 \mathrm{~m} 3 ; 213.4 \mathrm{~m} 3 ; 215.4 .1 \mathrm{~m} 2$; 217.1.1m2; 217.1.2m2; 217.1.3m2; 217.2.1m2; 219.1.3m2; 224.1.1m2; 224.3.2m2; 226.3m3; 229.2.2.m2. A unidade experimental foi um folíolo da terceira folha do terço apical. Realizaram-se a análise de variância e o teste de Duncan a $5 \%$ de probabilidade. A cv. IPA-5 apresentou baixa repelência; as progênies $206.1 .2 \mathrm{~m} 2,213.4 \mathrm{~m} 3$, 217.2.1m2 de baixa a média repelência; a cv. Caline IPA-6 e a progênie $229.2 .2 \mathrm{~m} 2$ média repelência e os genótipos L. hirsutum f. glabratum e L. hirsutum f. glabratum PI 134417 apresentaram alta repelência às fêmeas do ácaro T. urticae. A medição pelo teste de Weston e Snyder da repelência de genótipos ao ácaro foi satisfatória.

PALAVRAS-CHAVE: Solanum lycopersicum, Lycopersicon hirsutum, Tetranychus urticae.
ABSTRACT: The tomato crop is negatively affected by pests such as mite Tetranychus urticae (Koch) (Acari: Tetranychidae). This work aims to characterize the degree of repellency of tomato genotypes to T. urticae. The research was carried out at the Federal University of Lavras - UFLA at $23 \pm 2{ }^{\circ} \mathrm{C}$, from May to November 2001, in four tests with 19 treatments $(T)$ and 3 replicates (r): $6 \mathrm{~T}$ and $3 r, 19 \mathrm{~T}$ and $3 \mathrm{r}$, and $16 \mathrm{~T}$ and $5 r$. The T (genotypes) were two susceptible controls, Solanum lycopersicum IPA-5 and S. lycopersicum Caline IPA-6; two resistant controls, Lycopersicon hirsutumf. glabratum and L. hirsutumf. glabratum (PI 134417); progenies of S. lycopersicum $x$ Lycopersicon hirsutumf. glabratum : 184.1.1m2; $199.2 .2 \mathrm{~m} 2 ; 206.1 .2 \mathrm{~m} 2 ; 213.1 \mathrm{~m} 3 ; 213.4 \mathrm{~m} 3$; 215.4.1m2; 217.1.1m2; 217.1.2m2; 217.1.3m2; 217.2.1m2; 219.1.3m2; 224.1.1m2; 224.3.2m2; $226.3 \mathrm{~m} 3$ and 229.2.2. $\mathrm{m} 2$. The square root was one tomato leaflet from the third leave of the apical region of the plant. Data were analyzed by ANOVA and Duncan test at 5\%. The cultivar IPA-5 showed low repellency; the progenies 206.1.2m2, 213.4m 3 and 217.2.1m2 showed low to medium repellency; the cultivar Caline IPA-6 and the progenie 229.2.2m2 showed medium repellency and the genotypes L. hirsutum $f$. glabratum and L. hirsutumf. glabratum PI 134417 showed high repellency to $T$. urticae females. The measurement of the repellency of the tomato genotypes to the mite by the Weston and Snyder test was satisfactory.

KEYWORDS: Solanum lycopersicum, Lycopersicon hirsutum, Tetranychus urticae. 


\section{Introdução}

O ácaro rajado, Tetranychus urticae (Koch) (Acari: Tetranychidae), é responsável por significativas perdas em muitos cultivos ornamentais e hortícolas do mundo. No tomateiro, a espécie alimenta-se nas faces adaxial e abaxial dos seus folíolos, o que inibe a sua fotossíntese, podendo causar a sua queda (BARBOSA; FRANÇA, 1980; FLECHTMANN, 1989) e danos de pelo menos $13 \%$ (GONÇALVES et al., 1998).

$\mathrm{O}$ ácaro prefere plantas de determinadas espécies vegetais, o que faz parte da ecologia e evolução do espectro plantahospedeiro (AGRAWAL, 2000). Para a escolha da planta hospedeira, a fêmea de T. urticae, após realizar a picada de prova, etapa da fase de busca, pode se estabelecer, se alimentar $\mathrm{e}$, a depender da qualidade nutricional da planta, realizar sua oviposição ou se deslocar para longe (BRANDENBURG; KENNEDY, 1982; KENNEDY; SMITLEY, 1985; SABELIS, 1985; GRECO; PEREYRA; GUILLADE, 2006).

O processo de seleção realizado pelo ácaro poderia ser visto mais em termos de aceitação que de encontro da planta hospedeira (SABELIS, 1985). Nesse sentido, é de grande importância a utilização de métodos de análise de resistência de plantas a artrópodos, como o teste de repelência desenvolvido em folíolos de tomateiro por Weston e Snyder (1990), o qual mede a antixenose dos genótipos ao ácaro e permite determinação rápida e altamente quantitativa de diferentes graus de repelência de plantas a artrópodos. O teste foi utilizado por Gonçalves (1996) e Aragão (1998). O objetivo desta pesquisa foi caracterizar, pelo teste de Weston e Snyder (1990), a repelência de genótipos de tomateiro ao ácaro T. urticae.

\section{Material e Métodos}

Os experimentos foram realizados na Universidade Federal de Lavras, UFLA, em Minas Gerais, Brasil, à temperatura ambiente de $23 \pm 2{ }^{\circ} \mathrm{C}$, durante os meses de maio,.junho, setembro, outubro e novembro de 2001. Os tratamentos foram os seguintes:

- Genótipos: Solanum lycopersicum cv. IPA-5 [originado do genótipo IPA-3 x Pedigree Cal-J (INSTITUTO..., 1991a)] e S. lycopersicum cv. Caline IPA-6 [originado do genótipo IPA-3 x Pedigree PSX-76 (INSTITUTO..., 1991b)], testemunhas suscetíveis a artrópodos; Lycopersicon hirsutumf. glabratum PI 134417 e L. hirsutum f. glabratum C. H. Mull., testemunhas resistentes a artrópodos; progênies originadas do cruzamento de Solanum lycopersicum $\mathrm{x}$ Lycopersicon hirsutum $f$. glabratum : $184.1 .1 \mathrm{~m} 2$, 199.2.2m2, 206.1.2m2, 213.1m3, 213.4m3, 215.4.1m2, 217.1.1m2, 217.1.2m2, 217.1.3m2, 217.2.1m2, 219.1.3m2, 224.1.1m2, 224.3.2m2, 226.3m3 e 229.2.2.m2. Todos os materiais foram originários do Instituto Agronômico de Pernambuco- IPA. Os genótipos foram selecionados com relação às características agronômicas desejáveis, em campo e em telado, tendo sido observado menor ataque das plantas pela espécie Bemisia tabaci (Genn.) biótipo B (Hemiptera: Aleyrodidae).

Realizaram-se quatro experimentos: O primeiro, com 19 genótipos-tratamento e três repetições, englobou os ensaios de 1 a 3. O segundo foi organizado nos ensaios de 4 a 6 , com três repetições e seis genótipos-tratamento nos quais os ácaros percorreram as menores distâncias do primeiro ensaio. O terceiro experimento englobou os ensaios de 7 a 9 e foi realizado em três repetições com os 19 genótipos-tratamento do primeiro experimento. O quarto experimento, com cinco repetições e os 16 genótipos-tratamento selecionados no terceiro experimento, englobou os ensaios de 10 a 12.

Considerou-se unidade experimental o folíolo da terceira folha do terço apical da planta, região com maior teor de 2-tridecanona (LEITE, 1997). O autor, ao estudar plantas de S. lycopersicum cv. Santa Clara e de L. hirsutum f. glabratum (PI 134417) com 60, 90 e 120 dias em relação a sua resistência à espécie T. absoluta, verificou aumento dos teores de metilcetona nas plantas mais velhas. No presente trabalho, foram estudados folíolos de 99, 113, 64 e 96 dias, do primeiro ao quarto experimentos, respectivamente.

No pomar da UFLA, foram coletadas folhas de bananeiras das cvs. "Maçã", "Missouri", "Nanica" e "Prata", as quais foram acondicionadas em isopor até a montagem do experimento, realizado no mesmo dia da coleta. Dessas folhas foram coletadas, com pincel " 00 ", 10 fêmeas de T. urticae que foram transferidas para o centro de uma tachinha metálica, fixada na região central de um folíolo de tomateiro, disposto sobre placa de isopor com $1,5 \mathrm{~cm}$ de espessura previamente coberta por folha de papel branco de tamanho "A4" (210 mm x $297 \mathrm{~mm}$ ).

Aos 20, 40 e 60 minutos, foram medidas as distâncias médias percorridas pelas fêmeas na superfície do folíolo. Quando permaneceram sobre a tachinha, considerou-se zero mm a distância percorrida. Quando se encontravam na superfície foliar, mediu-se a distância do centro da tachinha (ponto zero) até o local onde estavam. Quando encontradas em algum ponto fora e além do folíolo, considerou-se como percorrida a distância do centro da tachinha até o bordo do folíolo, no sentido longitudinal.

Determinou-se a estimativa da distância média percorrida pelas 10 fêmeas no folíolo de tomateiro, nos tempos de observação e repetições do experimento. De acordo com Weston e Snyder (1990), para esse teste, quanto menor a distância média percorrida pelas fêmeas sobre os folíolos, maior se considera a repelência da planta a elas. Os dados foram submetidos à análise de variância e ao teste de Duncan a $5 \%$ de probabilidade.

\section{Resultados e Discussão}

As Tabelas de 1 a 4 contêm as estimativas das distâncias médias percorridas aos 20, 40 e 60 minutos pelas fêmeas de T. urticae nos genótipos de tomateiro e a interpretação do grau de sua repelência ao ácaro.

Os genótipos mais repelentes às fêmeas de T. urticae em todos os experimentos e tempos observados foram $L$. hirsutum f. glabratum (Tabelas 1, 3 e 4), e L. hirsutum f. glabratum (PI 134417) (Tabelas 1, 2, 3 e 4). O resultado era esperado para o acesso PI 134417, e concorda com o relatado por Gentile, Webb e Stoner (1969), Aina, Rodriguez e Knavel (1972), 
Tabela 1. Distância média estimada de três repetições percorrida por 10 fêmeas de Tetranychus urticae em genótipos de tomateiro aos 20 , 40 e 60 minutos Primeiro experimento, ensaios de 1 a 3, Lavras, MG, maio e junho de 2001.

\begin{tabular}{|c|c|c|c|}
\hline \multirow{2}{*}{$\begin{array}{l}\text { Tratamentos } \\
\text { (Genótipos) }\end{array}$} & \multicolumn{3}{|c|}{ Distância média $(\mathbf{m m}) \pm \mathbf{E P}$} \\
\hline & 20 minutos & 40 minutos & 60 minutos \\
\hline Solanum lycopersicum cv. IPA-5 & $17,02 \pm 2,58 \mathrm{a}^{(*)}$ & $18,00 \pm 3,00 \mathrm{ab}^{(*)}$ & $18,07 \pm 2,97 \mathrm{ab}^{(*)}$ \\
\hline Solanum lycopersicum cv. Caline IPA-6 & $9,27 \pm 1,24 \mathrm{e}^{(* * *)}$ & $9,03 \pm 0,97 f^{(* * *)}$ & $11,02 \pm 1,68 \mathrm{ef}^{(* * *)}$ \\
\hline Lycopersicon hirsutum f. glabratum & $2,12 \pm 0,45 \mathrm{f}^{(* * * *)}$ & $0,88 \pm 0,61 \mathrm{~g}^{(* * *)}$ & $1,35 \pm 0,80 \mathrm{~g}^{(* * * *)}$ \\
\hline Lycopersicon hirsutum f. glabratum PI 134417 & $0,42 \pm 0,42 \mathrm{f}^{(* * * *)}$ & $1,32 \pm 0,44 \mathrm{~g}^{(* * * *)}$ & $0,43 \pm 0,23 \mathrm{~g}^{(* * *)}$ \\
\hline Progênie de S. lycopersicum x L. hirsutum f. glabratum $184.1 .1 \mathrm{~m} 2$ & $10,43 \pm 0,69 \mathrm{bcde}^{(* * *)}$ & $13,63 \pm 2,03$ bcdef $^{(* *)}$ & $12,43 \pm 2,28 \operatorname{cdef}^{(* *)}$ \\
\hline Progênie de S. lycopersicum x L. hirsutum f. glabratum $199.2 .2 \mathrm{~m} 2$ & $9,63 \pm 1,02 \mathrm{de}^{(* * *)}$ & $10,87 \pm 0,58 \operatorname{def}^{(* * *)}$ & $11,58 \pm 1,38 \operatorname{def}^{(* * *)}$ \\
\hline Progênie de S. lycopersicum x L. hirsutum f. glabratum $206.1 .2 \mathrm{~m} 2$ & $13,23 \pm 1,10 \mathrm{abcde}^{(* *)}$ & $13,07 \pm 1,12$ bcdef $^{(* *)}$ & $13,03 \pm 1,41$ bcdef $^{(*)}$ \\
\hline Progênie de S. lycopersicum x L. hirsutum f. glabratum $213.1 \mathrm{~m} 3$ & $15,20 \pm 2,57 \mathrm{ab}^{(*)}$ & $15,43 \pm 0,93 \mathrm{abcd}^{(*)}$ & $16,47 \pm 1,63 \mathrm{abcd}^{(* *)}$ \\
\hline Progênie de S. lycopersicum x L. hirsutum f. glabratum $213.4 \mathrm{~m} 3$ & $10,90 \pm 0,32 \mathrm{bcde} \mathrm{e}^{(* * *)}$ & $10,93 \pm 0,61 \operatorname{def}^{(* * *)}$ & $11,00 \pm 0,64 \mathrm{ef}^{(* * *)}$ \\
\hline Progênie de S. lycopersicum x L. hirsutum f. glabratum $215.4 .1 \mathrm{~m} 2$ & $9,77 \pm 1,77 \mathrm{cde}^{(* * *)}$ & $10,35 \pm 1,24 \mathrm{ef}^{(* * *)}$ & $10,25 \pm 0,87 f^{(* * *)}$ \\
\hline Progênie de S. lycopersicum x L. hirsutum f. glabratum $217.1 .1 \mathrm{~m} 2$ & $13,60 \pm 1,93 \mathrm{abcde}^{(* *)}$ & $14,52 \pm 1,47$ bcde $^{(*)}$ & $15,67 \pm 1,75 \mathrm{abcde}^{(*)}$ \\
\hline Progênie de S. lycopersicum x L. hirsutum f. glabratum $217.1 .2 \mathrm{~m} 2$ & $12,57 \pm 3,37 \mathrm{abcde}^{(* *)}$ & $13,33 \pm 2,95 b c^{2} f^{(* *)}$ & $15,03 \pm 2,08 \mathrm{bcdef}^{(*)}$ \\
\hline Progênie de S. lycopersicum x L. hirsutum f. glabratum $217.1 .3 \mathrm{~m} 2$ & $13,03 \pm 0,30 \mathrm{abcde}^{(* *)}$ & $12,53 \pm 0,59 \mathrm{cdef}^{(* *)}$ & $12,23 \pm 0,54 \operatorname{def}^{(* * *)}$ \\
\hline Progênie de S. lycopersicum x L. hirsutum f. glabratum 217.2.1.m2 & $10,75 \pm 1,72 \mathrm{bcde}^{(* *)}$ & $13,05 \pm 1,10$ bcdef $^{(* * *)}$ & $12,70 \pm 1,63 \operatorname{cdef}^{(* *)}$ \\
\hline Progênie de S. lycopersicum x L. hirsutum f. glabratum $219.1 .3 \mathrm{~m} 2$ & $15,33 \pm 1,81 \mathrm{ab}^{(*)}$ & $19,42 \pm 0,81 a^{(*)}$ & $20,23 \pm 0,77 a^{(*)}$ \\
\hline Progênie de S. lycopersicum x L. hirsutum f. glabratum $224.1 .1 \mathrm{~m} 2$ & $16,47 \pm 0,64 \mathrm{a}^{(*)}$ & $16,33 \pm 0,50 \mathrm{abc}^{(*)}$ & $17,50 \pm 0,85 a b c{ }^{(*)}$ \\
\hline Progênie de S. lycopersicum x L. hirsutum f. glabratum $224.3 .2 \mathrm{~m} 2$ & $10,47 \pm 0,44 \mathrm{bcde}^{(* * *)}$ & $12,90 \pm 1,59 \mathrm{cdef}^{(* *)}$ & $14,17 \pm 2,22$ bcdef $^{(* *)}$ \\
\hline Progênie de S. lycopersicum x L. hirsutum f. glabratum $226.3 \mathrm{~m} 3$ & $15,10 \pm 1,05 \mathrm{abc}^{(*)}$ & $15,13 \pm 0,64 \mathrm{abcde}^{(*)}$ & $15,12 \pm 0,73$ bcdef $^{(* *)}$ \\
\hline Progênie de S. lycopersicum x L. hirsutum f. glabratum $229.2 .2 \mathrm{~m} 2$ & $14,77 \pm 1,13 \mathrm{abcd}^{(* *)}$ & $16,80 \pm 2,32 \mathrm{abc}^{(*)}$ & $16,03 \pm 1,56$ abcde ${ }^{(* *)}$ \\
\hline
\end{tabular}

Médias iguais na coluna não diferem pelo teste Duncan a $5 \%$ de probabilidade. ${ }^{(*)}$ Baixa repelência às fêmeas do ácaro Tetranychus urticae. ${ }^{(*)}$ Baixa a média repelência às fêmeas do ácaro Tetranychus urticae. ${ }^{(* * *)}$ Média repelência às fêmeas do ácaro Tetranychus urticae. ${ }^{\left({ }^{* * * *}\right)}$ Alta repelência às fêmeas do ácaro Tetranychus urticae.

Tabela 2. Distância média estimada de três repetições percorrida por 10 fêmeas de Tetranychus urticae em genótipos de tomateiro aos 20,40 e 60 minutos. Segundo experimento, ensaios de 4 a 6. Lavras, MG, junho de 2001.

\begin{tabular}{|c|c|c|c|}
\hline \multirow{2}{*}{$\begin{array}{l}\text { Tratamentos } \\
\text { (Genótipos) }\end{array}$} & \multicolumn{3}{|c|}{ Distância média $(\mathbf{m m}) \pm \mathbf{E P}$} \\
\hline & 20 minutos & 40 minutos & 60 minutos \\
\hline Solanum lycopersicum cv. IPA-5 & $15,70 \pm 1,21 b^{(*)}$ & $15,93 \pm 0,62 \mathrm{ab}^{(*)}$ & $16,30 \pm 0,85 b^{(*)}$ \\
\hline Solanum lycopersicum cv. Caline IPA-6 & $20,83 \pm 1,95 \mathrm{a}^{(* * *)}$ & $19,9 \pm 2,49 \mathrm{a}^{(*)}$ & $21,60 \pm 2,09 a^{(* * *)}$ \\
\hline Lycopersicon hirsutum f. glabratum PI 134417 & $0,43 \pm 0,18 \mathrm{c}^{(* * * *)}$ & $1,13 \pm 0,58 \mathrm{c}^{(* * * *)}$ & $0,50 \pm 0,29 \mathrm{c}^{(* * * *)}$ \\
\hline Progênie de S. lycopersicum x L. hirsutum f. glabratum $199.2 .2 \mathrm{~m} 2$ & $13,10 \pm 1,95 b^{(*)}$ & $13,23 \pm 1,88 b^{(*)}$ & $14,23 \pm 1,92 b^{(*)}$ \\
\hline Progênie de S. lycopersicum x L. hirsutum f. glabratum $213.4 \mathrm{~m} 3$ & $12,60 \pm 1,36 b^{(*)}$ & $14,33 \pm 1,22 b^{(*)}$ & $14,67 \pm 1,45 b^{(*)}$ \\
\hline Progênie de S. lycopersicum x L. hirsutum f. glabratum $215.4 .1 \mathrm{~m} 2$ & $12,77 \pm 1,16 b^{(*)}$ & $12,5 \pm 1,28 \mathrm{~b}^{(*)}$ & $12,30 \pm 0,57 b^{(*)}$ \\
\hline
\end{tabular}

Médias iguais na coluna não diferem pelo teste Duncan a $5 \%$ de probabilidade. ${ }^{(*)}$ Baixa repelência às fêmeas do ácaro Tetranychus urticae. ${ }^{(*)}$ Baixa a média repelência às fêmeas do ácaro Tetranychus urticae. ${ }^{(* *)}$ Média repelência às fêmeas do ácaro Tetranychus urticae. ${ }^{\left({ }^{* * * *}\right)}$ Alta repelência às fêmeas do ácaro Tetranychus urticae.

Rodriguez, Knavel e Aina (1972), Weston et al. (1989) e Silva, Lourenção e Moraes (1992), que o reportaram como resistente às espécies T. urticae, Tetranychus ludeni Zacher (Acari: Tetranychidae) e Tetranychus evansi Baker e Pritchard (Acari: Tetranychidae), devido à presença de altas concentrações das metilcetonas (2-UD) e 2-Tridecanona (2-TD) em seus folíolos e brotos, que, segundo Williams et al. (1980), Fery e Kennedy (1987), Eigenbrode e Trumble (1993), Weston et al. (1989) e Barbosa (1994), causam antibiose e antixenose em ácaros. Também se esperava que L. hirsutum f. glabratum, sem acesso definido, fosse repelente às fêmeas de T. urticae, por se tratar da mesma espécie do genótipo L. hirsutumf. glabratum (PI 134417), que supostamente apresente características botânicas semelhantes às deste.

Aina, Rodriguez e Knavel (1972) reportaram que um exsudato de tricomas glandulares do tomateiro resistente PI 251303, aplicado topicamente, foi altamente resistente (repelente) a T. urticae. Alguns desses exudatos, as 
Tabela 3. Distância média estimada de três repetições percorrida por 10 fêmeas de Tetranychus urticae em genótipos de tomateiro aos 20 , 40 e 60 minutos Terceiro experimento, ensaios de 7 a 9. Lavras, MG, setembro e outubro de 2001.

\begin{tabular}{|c|c|c|c|}
\hline \multirow{2}{*}{$\begin{array}{l}\text { Tratamentos } \\
\text { (Genótipos) }\end{array}$} & \multicolumn{3}{|c|}{ Distância média $(\mathbf{m m}) \pm \mathrm{EP}$} \\
\hline & 20 minutos & 40 minutos & 60 minutos \\
\hline Solanum lycopersicum cv. IPA-5 & $29,63 \pm 6,04 a b^{(*)}$ & $27,07 \pm 6,02 \operatorname{def}^{(*)}$ & $35,17 \pm 3,33 \mathrm{bc}^{(*)}$ \\
\hline Solanum lycopersicum cv. Caline IPA-6 & $28,97 \pm 5,44 \mathrm{ab}^{(*)}$ & $34,70 \pm 4,25 \mathrm{bcd}^{(*)}$ & $37,70 \pm 2,13 \mathrm{ab}^{(*)}$ \\
\hline Lycopersicon hirsutum f. glabratum & $0,43 \pm 0,43 \mathrm{~d}^{(* * * *)}$ & $0,67 \pm 0,12 h^{(* * *)}$ & $0,17 \pm 0,17 h^{(* * * *)}$ \\
\hline Lycopersicon hirsutum f. glabratum PI 134417 & $0,87 \pm 0,87 \mathrm{~d}^{(* * *)}$ & $0,30 \pm 0,30 h^{(* * *)}$ & $0,77 \pm 0,17 \mathbf{h}^{(* * *)}$ \\
\hline Progênie de S. lycopersicum x L. hirsutum f. glabratum $184.1 .1 \mathrm{~m} 2$ & $17,23 \pm 8,34 \mathrm{c}^{(* * *)}$ & $30,00 \pm 0,20 \operatorname{cdef}^{(*)}$ & $30,07 \pm 2,25 \mathrm{~cd}^{(*)}$ \\
\hline Progênie de S. lycopersicum x L. hirsutum f. glabratum $199.2 .2 \mathrm{~m} 2$ & $24,50 \pm 1,24 \mathrm{abc} c^{(*)}$ & $26,50 \pm 2,35 \operatorname{def}^{(*)}$ & $30,47 \pm 3,62 \mathrm{~cd}^{(*)}$ \\
\hline Progênie de S. lycopersicum x L. hirsutum f. glabratum $206.1 .2 \mathrm{~m} 2$ & $24,93 \pm 3,93 \mathrm{abc}{ }^{(*)}$ & $26,00 \pm 4,00 \mathrm{def}^{(*)}$ & $25,57 \pm 3,44 \mathrm{de}^{(* * *)}$ \\
\hline Progênie de S. lycopersicum x L. hirsutum f. glabratum $213.1 \mathrm{~m} 3$ & $19,20 \pm 3,67 \mathrm{bc}{ }^{(*)}$ & $40,00 \pm 0,00 \mathrm{ab}^{(*)}$ & $23,27 \pm 5,31 \mathrm{ef}^{(* * *)}$ \\
\hline Progênie de S. lycopersicum x L. hirsutum f. glabratum $213.4 \mathrm{~m} 3$ & $17,77 \pm 3,10 \mathrm{c}^{(* * *)}$ & $34,10 \pm 3,25 \mathrm{bcd}^{(*)}$ & $17,17 \pm 2,48 \mathrm{fg}^{(* * *)}$ \\
\hline Progênie de S. lycopersicum x L. hirsutum f. glabratum $215.4 .1 \mathrm{~m} 2$ & $25,07 \pm 2,78 \mathrm{abc}^{(*)}$ & $29,60 \pm 1,99 \operatorname{cdef}^{(*)}$ & $11,17 \pm 1,16 \mathrm{~g}^{(* * *)}$ \\
\hline Progênie de S. lycopersicum x L. hirsutum f. glabratum $217.1 .1 \mathrm{~m} 2$ & $20,63 \pm 1,28 \mathrm{abc}^{(*)}$ & $34,70 \pm 3,30 \mathrm{bcd}^{(*)}$ & $16,83 \pm 1,09 \mathrm{fg}^{(* * *)}$ \\
\hline Progênie de S. lycopersicum x L. hirsutum f. glabratum $217.1 .2 \mathrm{~m} 2$ & $16,40 \pm 2,33 \mathrm{c}^{(* * *)}$ & $17,17 \pm 1,33 \mathrm{~g}^{(* *)}$ & $22,57 \pm 1,15 \mathrm{fg}^{(* *)}$ \\
\hline Progênie de S. lycopersicum x L. hirsutum f. glabratum $217.1 .3 \mathrm{~m} 2$ & $25,67 \pm 4,12 \mathrm{abc}^{(*)}$ & $36,47 \pm 0,97 \mathrm{abc} c^{(*)}$ & $34,03 \pm 1,97 \mathrm{bc}{ }^{(*)}$ \\
\hline Progênie de S. lycopersicum x L. hirsutum f. glabratum 217.2.1.m2 & $30,93 \pm 3,07 \mathbf{a}^{(*)}$ & $40,20 \pm 2,18 \mathrm{ab}^{(*)}$ & $34,57 \pm 1,23 b c^{(*)}$ \\
\hline Progênie de S. lycopersicum x L. hirsutum f. glabratum $219.1 .3 \mathrm{~m} 2$ & $30,83 \pm 3,98 \mathrm{a}^{(*)}$ & $24,20 \pm 2,91 \mathrm{efg}^{(* * *)}$ & $43,20 \pm 1,89 \mathrm{a}^{(*)}$ \\
\hline Progênie de S. lycopersicum x L. hirsutum f. glabratum $224.1 .1 \mathrm{~m} 2$ & $25,40 \pm 1,02 \mathrm{abc}^{(*)}$ & $23,60 \pm 4,65 \mathrm{fg}^{(* * *)}$ & $21,10 \pm 3,04 \mathrm{ef}^{(* * *)}$ \\
\hline Progênie de S. lycopersicum x L. hirsutum f. glabratum $224.3 .2 \mathrm{~m} 2$ & $24,20 \pm 2,14 \mathrm{abc}^{(*)}$ & $32,07 \pm 2,68 \mathrm{bcdef}^{(*)}$ & $17,77 \pm 2,90 \mathrm{fg}^{(* * *)}$ \\
\hline Progênie de S. lycopersicum x L. hirsutum f. glabratum $226.3 \mathrm{~m} 3$ & $20,47 \pm 2,16 a b c^{(*)}$ & $43,60 \pm 4,16 \mathrm{a}^{(*)}$ & $21,27 \pm 0,41 \mathrm{ef}^{(* * *)}$ \\
\hline Progênie de S. lycopersicum x L. hirsutum f. glabratum $229.2 .2 \mathrm{~m} 2$ & $16,97 \pm 2,89 \mathrm{c}^{(* * *)}$ & $32,63 \pm 2,09 \mathrm{bcde}^{(*)}$ & $21,30 \pm 2,85 \mathrm{ef}^{(* * *)}$ \\
\hline
\end{tabular}

Médias iguais na coluna não diferem pelo teste Duncan a $5 \%$ de probabilidade. ${ }^{(*)}$ Baixa repelência às fêmeas do ácaro Tetranychus urticae. ${ }^{(* *)}$ Baixa a média repelência às fêmeas do ácaro Tetranychus urticae. ${ }^{(* * *)}$ Média repelência às fêmeas do ácaro Tetranychus urticae. ${ }^{(* * * *)}$ Alta repelência às fêmeas do ácaro Tetranychus urticae.

Tabela 4. Distância média estimada de cinco repetições percorrida por 10 fêmeas de Tetranychus urticae em genótipos de tomateiro aos 20,40 e 60 minutos. Quarto experimento, ensaios de 10 a 12. Lavras-MG, outubro e novembro de 2001.

\begin{tabular}{|c|c|c|c|}
\hline \multirow{2}{*}{$\begin{array}{l}\text { Tratamentos } \\
\text { (Genótipos) }\end{array}$} & \multicolumn{3}{|c|}{ Distância média $(\mathrm{mm}) \pm \mathbf{E P}$} \\
\hline & 20 minutos & 40 minutos & 60 minutos \\
\hline Solanum lycopersicum cv. IPA-5 & $21,58 \pm 1,39 \mathrm{bc}^{(*)}$ & $21,88 \pm 0,61 \mathrm{cde}^{(*)}$ & $22,24 \pm 0,50 \mathrm{abc}^{(*)}$ \\
\hline Solanum lycopersicum cv. Caline IPA-6 & $17,96 \pm 0,98 \mathrm{c}^{(*)}$ & $17,52 \pm 0,25 f^{(* * *)}$ & $16,58 \pm 0,58 \mathrm{e}^{(* * *)}$ \\
\hline Lycopersicon hirsutum f. glabratum & $0,00 \pm 0,00 \mathrm{~d}^{(* * * *)}$ & $0,16 \pm 0,16 \mathrm{~g}^{(* * * *)}$ & $0,00 \pm 0,00 f^{(* * * *)}$ \\
\hline Lycopersicon hirsutum f. glabratum PI 134417 & $0,46 \pm 0,33 \mathrm{~d}^{(* * * *)}$ & $0,48 \pm 0,48 \mathrm{~g}^{(* * * *)}$ & $0,10 \pm 0,10 f^{(* * * *)}$ \\
\hline Progênie de S. lycopersicum x L. hirsutum f. glabratum $184.1 .1 \mathrm{~m} 2$ & $21,91 \pm 2,43 \mathrm{abc}^{(*)}$ & $19,20 \pm 1,17 \operatorname{def}^{(* * *)}$ & $19,22 \pm 0,75 \mathrm{cde}^{(*)}$ \\
\hline Progênie de S. lycopersicum x L. hirsutum f. glabratum 199.2.2m2 & $18,24 \pm 1,04 \mathrm{c}^{(*)}$ & $22,48 \pm 1,57 \mathrm{bcd}{ }^{(*)}$ & $20,32 \pm 0,65 b c d^{(*)}$ \\
\hline Progênie de S. lycopersicum x L. hirsutum f. glabratum $206.1 .2 \mathrm{~m} 2$ & $19,80 \pm 1,35 \mathrm{bc}{ }^{(*)}$ & $17,80 \pm 0,49 \mathrm{ef}^{(* * *)}$ & $17,74 \pm 0,62 \mathrm{de}^{(* * *)}$ \\
\hline Progênie de S. lycopersicum x L. hirsutum f. glabratum $213.1 \mathrm{~m} 3$ & $19,78 \pm 2,03 \mathrm{bc}{ }^{(*)}$ & $20,84 \pm 1,71 \mathrm{cdef}^{(* *)}$ & $22,54 \pm 1,15 \mathrm{abc}^{(*)}$ \\
\hline Progênie de S. lycopersicum x L. hirsutum f. glabratum $213.4 \mathrm{~m} 3$ & $20,14 \pm 0,78 \mathrm{bc}{ }^{(*)}$ & $20,14 \pm 0,91 \operatorname{cdef}^{(* *)}$ & $23,84 \pm 1,72 a^{(*)}$ \\
\hline Progênie de S. lycopersicum x L. hirsutum f. glabratum $215.4 .1 \mathrm{~m} 2$ & $19,56 \pm 2,65 \mathrm{bc}{ }^{(*)}$ & $23,38 \pm 0,53 \mathrm{bcd}{ }^{(*)}$ & $22,06 \pm 1,91 \mathrm{abc}{ }^{(*)}$ \\
\hline Progênie de S. lycopersicum x L. hirsutum f. glabratum $217.1 .1 \mathrm{~m} 2$ & $17,72 \pm 1,47 \mathrm{c}^{(*)}$ & $20,54 \pm 0,76 \mathrm{cdef}^{(* *)}$ & $20,00 \pm 0,00 \mathrm{~cd}^{(* * *)}$ \\
\hline Progênie de S. lycopersicum x L. hirsutum f. glabratum $217.1 .2 \mathrm{~m} 2$ & $20,52 \pm 2,75 \mathrm{bc}^{(*)}$ & $25,96 \pm 1,23 \mathrm{ab}^{(*)}$ & $23,56 \pm 1,64 \mathrm{ab}^{(*)}$ \\
\hline Progênie de S. lycopersicum x L. hirsutum f. glabratum $224.1 .1 \mathrm{~m} 2$ & $26,34 \pm 1,95 \mathrm{a}^{(*)}$ & $25,10 \pm 1,45 \mathrm{bcd}^{(*)}$ & $22,54 \pm 2,09 \mathrm{abc}^{(*)}$ \\
\hline Progênie de S. lycopersicum x L. hirsutum f. glabratum $224.3 .2 \mathrm{~m} 2$ & $22,52 \pm 1,49 \mathrm{abc}^{(*)}$ & $27,90 \pm 2,16 a^{(*)}$ & $24,28 \pm 0,75 a^{(*)}$ \\
\hline Progênie de S. lycopersicum x L. hirsutum f. glabratum $226.3 \mathrm{~m} 3$ & $24,04 \pm 0,78 \mathrm{ab}^{(*)}$ & $24,10 \pm 0,46 b c^{(*)}$ & $24,28 \pm 0,31 a^{(*)}$ \\
\hline Progênie de S. lycopersicum $\times$ L. hirsutum f. glabratum $229.2 .2 \mathrm{~m} 2$ & $23,70 \pm 0,55 \mathrm{ab}^{(*)}$ & $20,52 \pm 2,33 \mathrm{cdef}^{(* *)}$ & $24,28 \pm 0,75 \mathrm{a}^{(*)}$ \\
\hline
\end{tabular}

Médias iguais na coluna não diferem pelo teste Duncan a $5 \%$ de probabilidade ${ }^{(*)}$ Baixa repelência às fêmeas do ácaro Tetranychus urticae. ${ }^{(* *)}$ Baixa a média repelência às fêmeas do ácaro Tetranychus urticae. ${ }^{(* *)}$ Média repelência às fêmeas do ácaro Tetranychus urticae. ${ }^{(* * *)}$ Alta repelência às fêmeas do ácaro Tetranychus urticae. 
metilcetonas, são presentes nos tricomas glandulares, que contém substâncias que, pela aderência e toxicidade, atuam na resistência a artrópodos. Tricomas não glandulares também podem contribuir com o processo. Chatzivasileiadis e Sabelis (1997) condicionaram a definição de quais plantas serão hospedeiras do ácaro à presença nelas dos tricomas foliares e dos metabólitos secundários, quer sejam tóxicos ou não, atraentes ou repelentes.

Carter e Snyder (1985) verificaram a correlação da densidade de tricomas glandulares do tipo IV dos genótipos de tomateiro L. hirsutum, e de híbridos F2 do cruzamento de $S$. lycopersicum com $L$. hirsutum com a resistência a $T$. urticae. Weston et al. (1989) verificaram correlação negativa entre a susceptibilidade dos ácaros e os níveis de 2-TD das glândulas de tricomas tipo VI. Gonçalves et al. (1998), constataram alta correlação negativa entre o teor da metilcetona nos folíolos dos genótipos e a distância média percorrida neles pelas fêmeas das espécies dos ácaros T. urticae e T. ludeni aos 60, 90 e 120 minutos, e concluíram que houve resistência mediada pelos níveis de 2-TD dos folíolos, expressa pela repelência de tomateiros Solanum a estas espécies.

Farrar Junior e Kennedy (1987), verificaram que os indivíduos de $T$. urticae visitaram e ovipositaram com maior frequência as áreas foliares não tratadas com as metilcetonas, que a 2-TD é mais tóxica, a 2-UD é menos tóxica e que não houve efeito sinergístico de ambas sobre o ácaro.

No presente trabalho, a cv. de S. lycopersicum IPA-5, suscetível, exibiu baixa repelência às fêmeas de $T$. urticae em todos os tempos e experimentos realizados. O comportamento da cv. Caline IPA-6 mostrou-se algumas vezes diferente do esperado: Aos 40 minutos do experimento 2, em todos os tempos do experimento 3 , e aos 20 minutos do experimento 4, exibiu baixa repelência, porém apresentou média repelência em todos os tempos do experimento 1 , aos 20 e aos 60 minutos do experimento 2, e aos 40 e 60 minutos do experimento 4 (Tabelas de 1 a 4). Portanto, julgamos mais adequado considerar como testemunha susceptível apenas a cv. IPA-5.

Considerando-se o que ocorreu mais vezes nos tempos de 20, 40 e 60 minutos do experimento 1, a cv. IPA-5 e as progênies 213.1.m3, 217.1.1m2, 217.1.2m2, 219.1.3m2 e $226.3 \mathrm{~m} 3$ apresentaram baixa repelência; as progênies $184.1 .1 \mathrm{~m} 2 \mathrm{e}$ 206.1.2m 2 exibiram de baixa a média repelência; a cv. Caline IPA-6 e as progênies 199.2.2m2, 213.4m3, 215.4.1m2, 217.1.3m2, 217.2.1m2, 224.3.2m 2 e 229.2.2m2 apresentaram média repelência às fêmeas de T. urticae (Tabela 1).

No segundo experimento, a cv. Caline IPA-6 exibiu, na maioria das vezes, média repelência às fêmeas do ácaro T. urticae, e os demais genótipos apresentaram resultados idênticos aos já descritos isoladamente nos tempos de 20, 40 e 60 minutos (Tabela 2).

No terceiro experimento, as cvs. IPA-5 e Caline IPA-6 e as progênies $184.1 .1 \mathrm{~m} 2,199.2 .2 \mathrm{~m} 2,206.1 .2 \mathrm{~m} 2,213.1 \mathrm{~m} 3$, 215.4.1m2, 217.1.1m2, 217.1.3m2, 217.2.1m2, 219.1.3m2, 224.3.2m2, 226.3m3 exibiram baixa repelência; as progênies $213.4 \mathrm{~m} 3,217.1 .2 \mathrm{~m} 2,224.1 .1 \mathrm{~m} 2$ e $229.2 .2 \mathrm{~m} 2$ apresentaram média repelência às fêmeas do ácaro T. urticae (Tabela 3).
No quarto experimento, a cv. IPA-5 e as progênies $184.1 .1 \mathrm{~m} 2,199.2 .2 \mathrm{~m} 2,213.1 \mathrm{~m} 3,213.4 \mathrm{~m} 3,215.4 .1 \mathrm{~m} 2$, $217.1 .2 \mathrm{~m} 2,224.1 .1 \mathrm{~m} 2,224.3 .2 \mathrm{~m} 2,226.3 \mathrm{~m} 3$ e $229.2 .2 \mathrm{~m} 2$ exibiram baixa repelência; a progênie $217.1 .1 \mathrm{~m} 2$ apresentou de baixa a média repelência; a cv. Caline IPA-6 e a progênie 206.1.2m2 exibiram média repelência às fêmeas de $T$. urticae (Tabela 4).

Considerando-se a maioria dos resultados dos quatro experimentos, a cv. IPA-5 apresentou baixa repelência; as progênies $206.1 .2 \mathrm{~m} 2,213.4 \mathrm{~m} 3,217.2 .1 \mathrm{~m} 2$ exibiram de baixa a média repelência; a cv. Caline IPA-6 e o genótipo 229.2.2m2 exibiram média repelência às fêmeas do ácaro T. urticae; os genótipos L. hirsutum f. glabratum e L. hirsutum f. glabratum PI 134417 apresentaram alta repelência às fêmeas do ácaro T. urticae.

As plantas exibem diferentes graus de aceitação ou de repelência aos ácaros, o que pode ocorrer entre cultivares dentro de famílias e até da mesma espécie botânica. No caso da repelência dos genótipos de tomate silvestres ao ácaro, podem estar envolvidos aspectos morfológicos da superfície foliar e especialmente dos tricomas; aspectos químicos, como a liberação de substâncias voláteis provenientes de metabólitos secundários da planta. Boom, Beek e Dicke (2003) e Dicke (1999) ampliaram a discussão do tema, quando relataram que as decisões de forrageamento de artrópodos são afetadas por fatores externos e internos como os ambientais abióticos, a presença de competidores ou de inimigos, a falta de alimento ou de certos nutrientes, de locais para a oviposição ou a aprendizagem dos artrópodos, possivelmente também envolvida no processo.

Dentre as Solanáceas, a espécie Nicotiana tabacum aceita muito bem o ácaro T. urticae, enquanto que as plantas da espécie Capsicum annuum o aceitam muito pouco (BOOM; BEEK; DICKE, 2003). No presente trabalho, a cv. de de S. lycopersicum IPA-5 exibiu baixa repelência, enquanto que a cv. Caline IPA-6 mostrou média repelência a T. urticae, o que pode ter ocorrido devido a possíveis diferenças na nutrição ou na composição das plantas.

Segundo Walling (2000), em tomateiro o herbívoro induz resposta direta de defesa da planta, caracterizada por aumento de compostos sinalizadores como jasmonato, etileno, salicilato e sistemin, os quais resultam de mudanças locais e sistêmicas, tais como a acumulação de inibidores de proteinase, polifenol oxidase, peroxidase, quitinases e síntese de calose. Portanto, as respostas diretas de defesa da planta de tomate ao ataque de ácaros induzem a estimulação de inibidores de proteinase. Li et al. (2002), relataram que o ataque do ácaro T. urticae induz o tomateiro a uma rápida resposta de defesa, pela regulação rápida do jasmonato. Kant et al. (2004), em estudo das respostas de defesa do tomateiro nos primeiros cinco dias do ataque de T. urticae, período no qual não houve oviposição, verificaram que os ácaros, quando se alimentaram, causaram lesões cloróticas nos tecidos das plantas. Quando as plantas de tomate foram infestadas por população do ácaro, ativaram as suas respostas de defesa diretas no primeiro, e as de defesas indiretas no terceiro dia do ataque. No quarto dia da infestação, 
a atividade inibidora de proteinase e particularmente a presença dos gens que regulam os níveis transcritos de salicilatos era ainda mantida.

Além disto, gens envolvidos no metabolismo dos fosfolipídeos foram potencialmente regulados no primeiro dia e aqueles do metabolismo secundário o foram apenas no quarto dia. Embora o aumento da regulação das enzimas envolvidas na biossíntese de monoterpenos e diterpenos já tivesse ocorrido no primeiro dia, o aumento significativo da emissão de terpenóides voláteis, apenas ocorreu no quarto dia da infestação das plantas pelo ácaro. Os autores concluíram que este aumento na produção de voláteis das plantas infestadas, coincidiu com a preferência olfatória do ácaro predador da espécie Phytoseiulus persimilis, o que indica que as plantas de tomate ativam suas defesas indiretas, expressas pela produção de compostos voláteis, para complementar as respostas de suas defesas diretas contra o ataque da espécie $T$. urticae.

No presente trabalho, o período de apenas 60 minutos foi suficiente para definir a aceitação ou a repelência dos genótipos de tomateiro a T. urticae. Kant et al. (2004), verificaram que há um aumento inicial nos níveis de vários gens de defesa de proteínas e metabólitos, ocorre a ativação simultânea de transdução de vias de sinalização de jasmonato, etileno e salicilato, como ocorreu em Arabidopsis e em fumo quando atacados por larvas de Lepidopteros e de Coleopteros (REYMOND et al., 2000; HALITSCHKE et al., 2003).

Segundo Strassner et al. (2002) a espécie T. urticae causou contínuos danos mecânicos à planta de tomate, o que provavelmente induziu a expressão do gen PR. Kant et al. (2004) julgam que existe a possibilidade de que o aumento dos níveis de transcrição dos gens PR seja uma resposta específica do tomateiro ao ácaro.

A medição da repelência de genótipos de tomateiro ao ácaro T. urticae pelo teste de Weston e Snyder, mostrou resultados satisfatórios no presente trabalho, o que concorda com o reportado por aqueles autores em 1990, como também por Gonçalves (1996) e por Aragão (1998). Para o aprofundamento das questões envolvidas e das respostas obtidas nesta pesquisa, sugerimos estudos morfológicos dos tricomas foliares; químicos, da composição foliar das plantas dos genótipos aqui pesquisados e de parâmetros biológicos de T. urticae, com ênfase no período e taxa de oviposição por fêmea e número de sua descendência por planta.

\section{Conclusões}

- Os genótipos mais repelentes ao ácaro são: L. hirsutum f. glabratum e L. hirsutum f. glabratum (PI 134417);

- A cv. Caline IPA-6 e a progênie 229.2.2m2 exibiram média repelência às fêmeas do ácaro T. urticae;

- As progênies 206.1.2m2, 213.4m3 e 217.2.1m2 exibiram de baixa a média repelência às fêmeas do ácaro T. urticae.

- A cv. IPA-5 apresentou baixa repelência às fêmeas do ácaro T. urticae;

- A medição pelo teste de Weston e Snyder da repelência de genótipos a T. urticae mostrou resultados satisfatórios.

\section{Agradecimentos}

Ao Dr. Venézio Felipe dos Santos pela análise estatística, a Áurea Araújo de Lacerda (in memorian) e a Dra. Desirée Maria Esmeraldino da Silva e a todos os outros que, direta ou indiretamente, contribuíram para a realização desta pesquisa.

\section{Referências}

AINA, O. J.; RODRIGUEZ, J. G.; KNAVEL, D. E. Characterizing resistance to Tetranychus urticae in tomato. Journal of Economic Entomology, Lanham, v. 65, p. 641-643, 1972.

AGRAWAL, A. A. Host-range evolution: adaptation and trade-offs in fitness of mites on alternative hosts. Ecology, Tempe, v. 81, p. 500508, 2000. http://dx.doi.org/10.1890/0012-9658(2000)081[0500:HR EAAT]2.0.CO;2

ARAGÃO, C. A. Tricomas foliares associados à resistência ao ácaro rajado Tetranychus urticae Koch. em linhagens de tomateiro com alto teor de 2-tridecanona nos folíolos. 1998. 71 f. Dissertação (Mestrado em Genética e Melhoramento de Plantas)-Universidade Federal de Lavras, Lavras, 1998.

BARBOSA, L. V. Controle genético e mecanismos de resistência em Lycopersicon spp. à traça-do-tomateiro (Scrobipalpuloides absoluta (Meyrick, 1917) (Lepidoptera: Gelechiidae). 1994. 71 f. Dissertação (Mestrado em Genética e Melhoramento de Plantas)-Universidade Federal de Lavras, Lavras, 1994.

BARBOSA, S.; FRANÇA, F. H. As pragas do tomateiro e seu controle. Informe Agropecuário, Belo Horizonte, v. 6, p. 37-40, 1980.

BOOM, C. E. M.; BEEK, T. A.; DICKE, M. Differences among plant species in acceptance by the spider mite Tetranichus urticae Koch. Journal of Applied Entomology, Berlin, v. 127, n. 3, p. 177-183, 2003. http://dx.doi.org/10.1046/j.1439-0418.2003.00726.x

BRANDENBURG, R. L.; KENNEDY, G. G. Intercrop relationships and spider mite dispersal in a corn/peanut agroecosystem. Entomologia Experimentalis et Applicata, Dordrecht, v. 32, p. 269-276, 1982. http://dx.doi.org/10.1111/j.1570-7458.1982.tb03217.x

CARTER, C. D.; SNYDER, J. C. Mite responses in relation to trichomes of Lycopersicon esculentum $\mathrm{x}$ Lycopersicon hirsutum $\mathrm{F} 2$ hybrids. Euphytica, Wageningen, v. 34, p. 177-185, 1985. http:// dx.doi.org/10.1007/BF00022877

CHATZIVASILEIADIS, E. A.; SABELIS, M. W. Toxicity of methyl ketones from tomato trichomes to Tetranychus urticae Koch. Experimental Apllied of Acarology, v. 21, p. 473-484, 1997. http://dx.doi.org/10.1023/A:1018436113892

DICKE, M. Are herbivore-induced plant volatiles reliable indicators of herbivore identity to foraging carnivorous arthropods? Entomologia Experimentalis et Applicata, Dordrecht, v. 91, n. 1, p. 131-142, 1999. http://dx.doi.org/10.1046/j.1570-7458.1999.00475.x

EIGENBRODE, S. D.; TRUMBLE, J. T. Antibiosis to beet armyworm (Spodoptera exigua) in Lycopersicon accessions. Horticultural Science, v. 28, p. 932-934, 1993

FARRAR JUNIOR, R. R.; KENNEDY, G. G. 2-Undecanone, a constituent of the glandular trichomes of Lycopersicon hirsutum $\mathrm{f}$. glabratum: effects on Heliothis zea and Manduca sexta growth and survival. Entomologia Experimentalis et Applicata, Dordrecht, v. 43, p. 17-23, 1987.

FERY, R. L.; KENNEDY, G. G. Genetic analysis of 2-Tridecanone concentration, leaf trichome characteristics and tobacco hornworm resistance in tomato. Journal of the American Society for Horticultural Science, Alexandria, v. 112, p. 886-891, 1987. 
FLECHTMANN, C. H. W. Ácaros de importância agrícola. São Paulo: Nobel, 1989. 189 p.

GENTILE, A. G.; WEBB, R.; STONER, A. K. Lycopersicon and Solanum resistant to the carmine and the two-spotted spider mite. Journal of Economic Entomology, Lanham, v. 62, p. 834-836, 1969.

GONÇALVES, M. I. F. Variação do teor de 2-tridecanona em folíolos de tomateiro e sua relação com a resistência a duas espécies de ácaros do gênero Tetranychus. 1996. 63 f. Dissertação (Mestrado em Genética e Melhoramento de Plantas)-Universidade Federal de Lavras, Lavras, 1996.

GONÇALVES, M. I. F. et al. Variation of 2-Tridecanone level in tomato plant leaflets and resistance to two mite species (Tetranychus sp.). Euphytica, Wageningen, v. 104, p. 33-38, 1998. http://dx.doi. org/10.1023/A:1018611514287

GRECO, N. M.; PEREYRA, P. C.; GUILLADE, A. Host-plant acceptance and performance of Tetranychus urticae (Acari, Tetranychidae). Journal of Applied Entomology, Berlin, v. 130, n. 1, p. 32-36, 2006. http://dx.doi.org/10.1111/j.1439-0418.2005.01018.x

HALITSCHKE, R. et al. Molecular interactions between the specialist herbivore Manduca sexta (Lepidoptera, Sphingidae) and its natural host Nicotiana attenuata. VI. Microarray analysis reveals that most herbivore-specific transcriptional changes are mediated by fatty acid-amino acid conjugates. Plant Physiology, Minneapolis, v. 131, n. 4, p. 1894-1902, 2003. http://dx.doi.org/10.1104/pp.102.018184

INSTITUTO AGRONÔMICO DE PERNAMBUCO - IPA. IPA-5 - Cultivar de tomateiro recomendada pelo IPA. Recife: IPA, 1991a. 1 p. (IPA - Divulga, n. 41).

INSTITUTO AGRONÔMICO DE PERNAMBUCO - IPA. Caline IPA-6 - Cultivar de tomateiro recomendada pelo IPA. Recife: IPA, 1991b. 1 p. (IPA Divulga, n. 42).

KANT, M. R. et al. Differential timing of spider mite-induced direct and indirect defenses in tomato plants. Plant Physiology, Minneapolis, v. 135, p. 483-495, 2004. http://dx.doi.org/10.1104/pp.103.038315

KENNEDY, G. G.; SMITLEY, D. R. Dispersal. In: HELLE, W.; SABELIS, M. Spider mites, their biology, natural enemies and control. Amsterdam: Elsevier, 1985. p.233-242.

LEITE, G. L. D. Efeito da idade, parte do dossel e níveis de adubação NK na resistência de Lycopersicon hirsutum f. glabratum à Tuta absoluta. 1997. 40 f. Tese (Mestrado em Entomologia)-Universidade Federal de Viçosa, Viçosa, 1997.
LI, C. et al. Resistance of cultivated tomato to cell content-feeding herbivores is regulated by the octadecanoid-signaling pathway. Plant Physiology, Minneapolis, v. 130, n. 1, p. 494-503, 2002. http://dx.doi.org/10.1104/pp.005314

REYMOND, P. et al. Differential gene expression in response to mechanical wounding and insect feeding in Arabidopsis. Plant Cell, Rockville, v. 12, p. 707-719, 2000.

RODRIGUEZ, J. G.; KNAVEL, D. E.; AINA, O. J. Studies in the resistance of tomatoes to mites. Journal of Economic Entomology, Lanham, v. 65, p. 50-53, 1972.

SABELIS, M. W. Reproductive strategies. In: HELLE, W.; SABELIS, M. Spider mites, their biology, natural enemies and control Amsterdam: Elsevier, 1985. p.265-278.

SILVA, C. A. D.; LOURENÇÃO, A. L.; MORAES, G. J. Resistência de tomateiros ao ácaro vermelho Tetranychus evansi Baker \& Pritchard (Acari: Tetranychidae). Anais da Sociedade Entomológica do Brasil, Jaboticabal, v. 21, p. 147-156, 1992.

STRASSNER, J. et al. Characterization and cDNA-microarray expression analysis of 12- oxophytodienoate reductases reveals differential roles for octadecanoid biosynthesis in the local versus the systemic wound response. The Plant Journal, Oxford, v. 32, n.4, p. 585-601, 2002. http://dx.doi.org/10.1046/j.1365-313X.2002.01449.x

WALLING, L. L. The myriad plant responses to herbivores. Journal of Plant Growth Regulation, New York, v. 19, p. 195-216, 2000.

WESTON, P. A. et al. Trichome secretion composition, trichome densities, and spider mite resistance of ten accessions of Lycopersicon hirsutum. Journal of the American Society of the Horticultural Science, Alexandria, v. 114, p. 492-498, 1989.

WESTON, P. A.; SNYDER, J. C. Thumbtack Biossay: a quick method for measuring plant resistance to twospotted spider mites (Acari: Tetranychidae). Journal of Economic Entomology, Lanham, v. 2, p. 500-504, 1990.

WILLIAMS, W. G. et al. 2-Tridecanone: a naturally occurring insecticide from the wild tomato Lycopersicon hirsutum $\mathrm{f}$. glabratum. Science, v. 207, p. 888-889, 1980. http://dx.doi. org/10.1126/science.207.4433.888 